\title{
Synthesis of a DNA-targeting nickel (II) complex with testosterone thiosemicarbazone which exhibits selective cytotoxicity towards human prostate cancer cells (LNCaP)
}

\begin{abstract}
Testosterone thiosemicarbazone, L and its nickel (II) complex 1 were synthesized and characterized by using FTIR, CHN, (1)H NMR, and X-ray crystallography. X-ray diffraction study confirmed the formation of $\mathrm{L}$ from condensation of testosterone and thiosemicarbazide. Mononuclear complex 1 is coordinated to two Schiff base ligands via two imine nitrogens and two tautomeric thiol sulfurs. The cytotoxicity of both compounds was investigated via MTT assay with cisplatin as positive reference standard. L is more potent towards androgendependent LNCaP (prostate) and HCT 116 (colon). On the other hand, complex 1, which is in a distorted square planar environment with L acting as a bidentate NS-donor ligand, is capable of inhibiting the growth of all the cancer cell lines tested, including PC-3 (prostate). It is noteworthy that both compounds are less toxic towards human colon cell CCD-18Co. The intrinsic DNA binding constant $(\mathrm{Kb})$ of both compounds were evaluated via UV-Vis spectrophotometry. Both compounds showed $\mathrm{Kb}$ values which are comparable to the reported $\mathrm{Kb}$ value of typical classical intercalator such as ethidium bromide. The binding constant of the complex is almost double compared with ligand L. Both compounds were unable to inhibit the action topoisomerase I, which is the common target in cancer treatment (especially colon cancer). This suggest a topoisomerase I independent-cell death mechanism.
\end{abstract}

Keywrod: Cytotoxicity; DNA; Nickel; Schiff base 